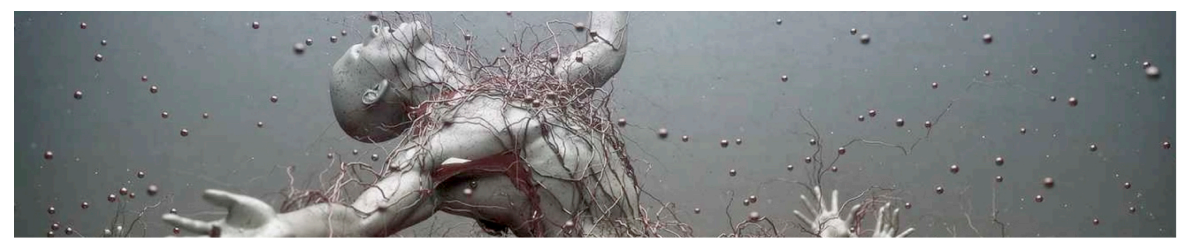

\title{
Comunicación y Mediación social para el cam- bio. Proyecto Gestión Integral de Cuencas con enfoque participativo. Casos: Ríos Pao y Unare
}

\section{Communication and Social Mediation for the Change. Integrated Watershed Management Project with Participatory Approach. Case: Rivers Pao and Unare}

\author{
María Ángela Flores ${ }^{(*)}$ \\ Universidad de Carabobo, \\ Facultad de Ciencias Económicas y Sociales - Venezuela \\ mariangelafp@hotmail.com \\ Aracelis Arana ${ }^{(* *)}$ \\ Universidad Pedagógica Experimental Libertador, \\ Pedagógico de Maracay - Venezuela \\ aracelisarana3@gmail.com \\ Esmeya Díaz de Mariño ${ }^{(* * *)}$ \\ Universidad de Carabobo, \\ Facultad de Ciencias Económicas y Sociales - Venezuela \\ esmeyadiazm@gmail.com \\ Abstract \\ The work presented is intended to \\ refer the process followed in the
}

Resumen

El trabajo que se presenta tiene por objeto referir el proceso seguido en la 
construcción de un Modelo de Mediación Comunicacional concebido para llevar a cabo la acción social transformadora a través de la comunicación pública de la información recabada durante la ejecución del proyecto de Gestión Integral de Cuencas con enfoque participativo, casos Ríos Pao y Unare, que se desarrolló con la finalidad de analizar el estado de dichas cuencas, fomentar su conservación y mejoramiento local en el marco del desarrollo endógeno y sustentable. Metodológicamente se realizó una investigación documental y de campo de tipo descriptivo, diseñada en tres fases: primera, desarrollo del modelo comunicacional que integra el trabajo y la dinámica de la red de investigadores, actores claves, organizaciones y poblaciones involucradas; segunda, estructuración de la información en diversos materiales comunicacionales: boletines, desplegables, calendarios, notas de prensa, trabajos especiales, micros de radio y una página Web; y tercera: la compilación de la experiencia. Entre los resultados a destacar tenemos: a) el impacto comunicacional en las comunidades está asociado a la identificación del proyecto y el uso de imágenes reconocidas por los pobladores acompañadas de un lenguaje sencillo; b) la presencia en los medios ha permitido dar cuenta de la interacción Estado-universidadcomunidad y propiciar el conocimiento de las cuencas en estudio. Finalmente el componente comunicacional permitió evidenciar el proceso de aprendizaje colectivo y la evolución de la conformación de la conciencia pública en la gestión integral de cuencas; asimismo, su compilación posibilita replicar la experien- construction of a Mediation Model of Communicational designed to carry out transformational social action through the public communication of the information gathered during the execution of the draft Comprehensive Watershed Management with participatory approach, Pao and Unare cases Rivers, which was developed in order to analyze the status of such basins, promote conservation and local improvement under the endogenous and sustainable development. Methodologically documentary and descriptive field research, designed in three phases was performed: first, development communication model that integrates work and the dynamics of the network of researchers, stakeholders, organizations and populations involved; Second, structuring information in various communication materials: newsletters, brochures, calendars, press releases, special papers, radio micros and a Web page; and third: the compilation of the experience. Among the outstanding results we have: a) the communication impact on communities is associated with project identification and use of images recognized by villagers accompanied by simple language; b) the presence in the media has allowed the interaction to account for stateuniversity-community and promoting awareness of the watershed study. Finally, the communication component allowed evidence the collective learning process and the evolution of shaping public con-

Mediaciones Sociales, № 13, 2014, pp. 131-159. ISSN electrónico:

1989-0494.

DOI: http://dx.doi.org/10.5209/rev_MESO.2014.n13.49435 
cia en otros contextos.

Palabras claves: Gestión de cuencas, comunicación pública, mediación y participación. sciousness in integrated river basin management; also enables compilation replicate the experience in other contexts.

Keywords: Watershed management, public communication, mediation and participation.

Sumario: 1. Introducción; 2. Objetivos; 3. Marco Referencial; 3.1. Evolución de los enfoque en la gestión de cuencas; 3.2. Comunicación y acción social transformadora; 3.2.a. Comunicación para la participación; 3.2.b. Mediación comunicacional y aprendizaje social; 3.3. Líneas de acción para una estrategia comunicacional con enfoque participativo; 4. Metodología; 4.1. Proceso seguido en el diseño del Modelo Comunicacional; 4.2. Modelo Comunicacional GICEP; 5. Recuperación de la experiencia vivida; 6. Conclusiones; Bibliografía.

\section{INTRODUCCIÓN}

En el marco del Proyecto: Gestión Integral de Cuencas con un enfoque participativo, Casos: Ríos Pao y Unare, se prevé la comunicación como mediador social de la información y los cambios que se van desarrollando en los colectivos a lo largo de su ejecución; en tal sentido, para dar a conocer los avances del mismo se diseñó una estrategia comunicacional cuyas fases se integraron en un modelo general que se ha venido ejecutando progresivamente desde el año 2009 (Arana, Flores y Díaz, 2009). El trabajo que se reporta tiene como propósito esbozar la evolución del modelo comunicacional empleado y los aspectos considerados para la transformación social a partir de un proceso de análisis reflexivo de las actividades realizadas con las comunidades involucradas, en este caso particular las comunidades correspondientes a las Cuenca de los Ríos Pao y Unare, ubicadas en Venezuela, concretamente en los Municipios Libertador del Estado Carabobo y Cajigal del Estado Anzoátegui respectivamente.

\section{OBJetivos}

a) Definir las principales líneas de acción estratégica que sustentaron el desarrollo del modelo comunicacional de mediación social objeto de estudio.

Mediaciones Sociales, № 13, 2014, pp. 131-159. ISSN electrónico: $1989-0494$.

DOI: http://dx.doi.org/10.5209/rev_MESO.2014.n13.49435 
b) Describir el proceso seguido en el diseño del modelo comunicacional de mediación social empleado.

c) Reconstruir, a partir de un proceso de reflexión de la experiencia vivida, los momentos implicados en la concreción del proceso comunicacional diseñado.

d) Analizar los procesos de mediación social alcanzados.

\section{MARCO REFERENCIAL}

\subsection{Evolución de los enfoque en la gestión de cuencas}

Los alcances del manejo de cuencas en la década de los setenta, evolucionaron desde un enfoque orientado puramente a la captación de agua a otros niveles más complejos como los de protección de los recursos naturales y mitigación del efecto de fenómenos naturales extremos, control de erosión, control de la contaminación, conservación de suelos y rehabilitación, recuperación de zonas degradadas, para luego pasar a los de mejoramiento de producción, primero forestal y de pastos, y posteriormente agrícola, agroforestería o agrosilvopastoril en forma combinada.

En el Primer Congreso de Manejo de Cuencas Hidrográficas (REDLACH - FAO, 1990: 6) realizado en la ciudad de Concepción (Chile), se acepta como definición de manejo integral de cuencas: "gestión que se realiza en un sistema hidrográfico, para aprovechar y proteger los recursos naturales que ofrece, con el fin de obtener una producción óptima y sostenida,... mejorando la calidad de vida de sus pobladores y manteniendo el equilibrio ambiental".

En épocas más recientes, esta expansión del concepto original de manejo de cuencas lo ha hecho extensivo al manejo integrado de los recursos naturales de una cuenca, y por último a la gestión ambiental integrada. Los nuevos enfoques están dirigidos a generar propuestas desde la base hacia arriba, tal como lo señala (Lozano, 2004) se trata de una acción concertada entre entidades gubernamentales y sociedad civil, buscando atender a los sectores más vulnerables de la población.

De acuerdo con FODEPAL (2004), el enfoque de gestión integrada de cuencas hidrográficas, con sus distintos niveles operativos por aproximaciones sucesivas, cuenca-subcuenca-microcuenca, permite compatibilizar

Mediaciones Sociales, № 13, 2014, pp. 131-159. ISSN electrónico: $1989-0494$.

DOI: http://dx.doi.org/10.5209/rev_MESO.2014.n13.49435 
las prioridades de los distintos sectores involucrados en cada región, el aumento de la producción con la conservación de los recursos naturales y mejorar la calidad de vida de sus habitantes, sin deteriorar el medio ambiente. En definitiva, se permite promover un desarrollo sostenible como fin último para lograr el crecimiento económico, la equidad social y la conservación del medio ambiente (p.12).

De acuerdo con (Dourojeanni, 2010:12), los principios que subyacen en las estrategias de gestión Integrada de Recursos Hídricos son:

- Complementariedad: La implementación de la Estrategia se hará en forma gradual. Una de sus metas es armonizar los objetivos y las líneas de acción de los diversos servicios públicos.

- Descentralización: La Estrategia se abocará a crear nuevas capacidades de gestión descentralizada sobre territorios delimitados por razones naturales manteniendo la armonía con los intereses de las autoridades a cargo de los territorios delimitados por razones político administrativos.

- Valoración de la Diversidad País: La implementación de la Estrategia pondrá en valor la diversidad natural y cultural, generando acciones asociadas al carácter singular.

- Desarrollo Territorial Sustentable: Las intervenciones en el territorio de las cuencas se hará promoviendo el uso sustentable de los elementos y recursos naturales.

- Prevención de Conflictos: La Estrategia se abocará al encuentro de formas de complementación público-privada, que aseguren mayores niveles de participación.

- Participación de la Sociedad Civil: El involucramiento de la sociedad civil en las decisiones en torno a las intervenciones que se realicen en el territorio, constituye un aporte significativo a la Democracia Participativa y la llamada gobernabilidad.

- Acceso a la Información y al Conocimiento: Tanto la calidad de la información, como el nivel de conocimiento que se tiene del territorio constituye uno de los pilares para la toma de decisiones acertadas. El acceso

Mediaciones Sociales, № 13, 2014, pp. 131-159. ISSN electrónico: 1989-0494.

DOI: http://dx.doi.org/10.5209/rev_MESO.2014.n13.49435 
tanto público como privado a la información, da garantías de generar procesos transparentes y creíbles.

En la actualidad, los diversos enfoques que se desarrollan en el campo de la gestión integral de cuencas coinciden en varios aspectos de fundamental importancia, como lo son:

a) La sostenibilidad de los recursos naturales

b) La participación de los habitantes residentes en la toma de decisiones

c) La articulación entre los distintos entes gubernamentales y los actores claves

d) La prevención de riesgo

e) El beneficio productivo

f) La equidad

g) La información y comunicación como herramientas para el empoderamiento de las comunidades.

En este sentido, las autoras asumieron la comunicación y la información como un eje transversal al modelo de gestión, debido a su importancia como herramienta mediadora en los procesos de formación y transformación comunitaria, tal como lo evidencian algunos estudios realizados, que se mencionan seguidamente.

\subsection{Comunicación y acción social transformadora}

La revisión documental realizada muestra una preocupación en el ámbito regional latinoamericano por la protección de los recursos acuíferos y los sistemas de cuencas por donde circulan, en la que se manifiesta también una tendencia hacia la gestión de estos recursos con la implicación de la población, es decir, la sustentabilidad de los procesos de gestión de las cuencas depende en gran medida de la participación comprometida de los habitantes de estos sectores, por cuanto ellos se transforman en actores claves para fomentar, desarrollar y monitorear la participación social en los procesos de gestión y conservación de los recursos naturales.

Lo anterior se vincula con la Agenda 21, pues a través de su aplicación se toma en consideración cuatro criterios: a) que exista un proceso, b) una política en la que se aplique lo que se decida, c) un producto, es decir,

Mediaciones Sociales, № 13, 2014, pp. 131-159. ISSN electrónico: 1989-0494.

DOI: http://dx.doi.org/10.5209/rev_MESO.2014.n13.49435 
un cambio que se pueda apreciar con el tiempo y, de manera transversal, d) una participación de todos los sectores de la ciudadanía del municipio; Esto es lo que se denomina, según Arnanz, Belmonte, García, Garrido y Heras (2007), las “4P”: proceso, política, producto y participación (p. 1).

La Agenda 21 surge en la "Cumbre de la Tierra", celebrada en Río de Janeiro en 1992, y supone una estrategia promovida por las Naciones Unidas de cara al desarrollo sustentable en el planeta (PNUMA, 2005). La lógica que guía este instrumento tiene que ver con el fomento de la sustentabilidad global a partir de la actuación local (en los municipios, las regiones, las comunidades, entre otras). La implementación de esta agenda, se inicia con la realización de un diagnóstico sobre la realidad local, crear foros de participación ciudadana, un Plan de Acción Local y un sistema de indicadores; no obstante, el fomento de la participación no se logra sino se cuenta con una estrategia de comunicación (Arnanz y otros, 2007: 2), sin información es difícil participar, de manera que la información y la comunicación son elementos claves para la participación, es importante también un acceso continuo y confiable a las fuentes de información, así como también que existan mecanismos de comunicación entre los diferentes niveles de participación de la sociedad.

Asimismo, de acuerdo con Arnanz y otros (2007:3) integrantes de Observatorio Internacional de Ciudadanía y Medio Ambiente Sostenible (CIMAS), es fundamental el conocimiento de las redes locales para poder llevar a cabo una labor de implicación ciudadana en el desarrollo sustentable de una comunidad: "el acercamiento a los centros neurálgicos donde se aglutina la acción de los distintos grupos o actores locales, la integración en su actividad cotidiana y la observación participante, permiten manejar las claves de las redes sociales y estar en condiciones de fomentar su participación en el proceso, así como, adecuar los mensajes y los canales de comunicación.

\section{2.a. Comunicación para la participación}

De acuerdo con Ridder, Mostert y Wolters (2005) dentro de la comunicación existen diferentes niveles de participación: a) Información, tiene que ver con proporcionar acceso a la información y difundirla activamente, b) Consulta, se refiere a la posibilidad de que el público pueda realizar comentarios por escrito, o mediante entrevistas personales o encuestas y c)

Mediaciones Sociales, № 13, 2014, pp. 131-159. ISSN electrónico: 1989-0494.

DOI: http://dx.doi.org/10.5209/rev_MESO.2014.n13.49435 
Participación activa, implica un papel más activo del público: charlas con las autoridades, colaborar en determinadas agendas políticas, incorporarse en la toma de decisiones, comprometerse en el desarrollo de soluciones y en general implicarse en la gestión de la cuenca, lo cual requiere de un aprendizaje social basado en el diálogo entre todas las partes interesadas. Esta forma de participación hace referencia, según Hernández (2010), a un cambio en los mecanismos de gobernación para que den cabida a los intereses de una amplia mayoría de la población, va más allá de lo meramente asistencial, informativo o consultivo, para alcanzar a una población que tiene el derecho de decidir sobre lo que le afecta en su vida en común, es una manera de ejercer el gobierno basada en actitudes políticas que tienen como medio y como fin gobernar con los ciudadanos y no solo para los ciudadanos.

En este sentido, es necesario que los individuos se organicen, tal como se señala en el Proyecto Nacional Simón Bolívar (República Bolivariana de Venezuela, 2007-2013:14):

...para lograr las ventajas que otorga la asociación cooperativa, es decir, transformar su debilidad individual en fuerza colectiva, teniendo en cuenta que el establecimiento de la organización no implicará menoscabo de la independencia, autonomía, libertad y poder originario del individuo, dicha unión debe estar orientada a poner de relieve los elementos en común, que permitan construir una comunidad.

Esta participación de la sociedad civil, desde la perspectiva de Bustos (2006:22 ) ... "actúa en lo local y en lo global al mismo tiempo, esta coexistencia le concede un carácter central al individuo y al grupo", de manera que de acuerdo con Arana (2008), se puede interpretar que se asiste a un nuevo enfoque dialógico entre lo individual y lo grupal, donde se establece una sinergia reestructurante entre el grupo y el individuo, mediante una dinámica recursiva, en donde las acciones individuales coadyuvan al logro de las metas comunes del grupo, y a su vez, estos logros del colectivo benefician la actividad individual, creándose una espiral de crecimiento progresivo, que conduce a la comunidad y a sus habitantes a niveles de mayor organización social y mejor calidad de vida.

\section{2.b. Mediación comunicacional y aprendizaje social}

Mediaciones Sociales, № 13, 2014, pp. 131-159. ISSN electrónico:

1989-0494.

DOI: http://dx.doi.org/10.5209/rev_MESO.2014.n13.49435 
El aprendizaje social significa el aprendizaje de todos los actores sociales comprometidos en gestionar asuntos en los que tienen intereses comunes (Ridder y otros, 2005), en otras palabras, cuando es necesario llegar a un entendimiento compartido de los temas a tratar y de las posibles soluciones. Para ello es necesario, según Hernández, (2010), que los responsables que acompañan el proceso de participación adopten una actitud de aprendizaje dialógico en el que "ni hay nadie que sepa todo, ni nadie hay que no sepa nada" (Freire, citado por Hernández, ob cit). Esa actitud abierta llevará a los responsables de la participación a escuchar a todos y cada uno de los que participan y a gestionar "comunicativamente" las distintas valoraciones y concepciones, así como, las opiniones diferentes y a veces divergentes que aportan los participantes, sus organizaciones o las administraciones.

Por consiguiente, para gestionar conjuntamente el desarrollo sustentable de una cuenca en particular, no sólo es necesario aprender los aspectos técnicos de la gestión de la cuenca de los ríos, sino que también deben reconocerse las preocupaciones y los puntos de vista de todos los actores sociales; así como, llegar a un acuerdo y contar con recursos conjuntos para implementar este acuerdo. En este sentido, la comunicación pública llevada a cabo estratégicamente puede brindar beneficios convenientes al colectivo implicado en la gestión integral, desplegando la capacidad para que los diversos actores puedan poner en práctica las acciones para el cambio y gestionando el diálogo entre los múltiples actores a fin de desarrollar en ellos el sentido de apropiación de los problemas y de las soluciones para que las acciones se sostengan en el tiempo (Hesselink, Goldstein, van Kempen, Garnett y Dela, 2007).

Asimismo, se requiere que el sistema de asesoramiento, mediación y acompañamiento externo sepa gestionar las dinámicas que aparecen durante el proceso de participación. Lo cual es, en sintonía con Hernández (2010), la tarea fundamental del mediador social, del técnico o experto en participación, gestionar la dinámica de participación de modo que llegue a buen puerto. Todo ello con un único objetivo, obtener la convergencia de los mismos, consiguiendo una cierta unidad entre ellos, algunos acuerdos aunque sean parciales, la puesta en común, o por lo menos generar tolerancia ante la divergencia y la diversidad incluso ambivalencia de los resultados.

Mediaciones Sociales, № 13, 2014, pp. 131-159. ISSN electrónico: 1989-0494.

DOI: http://dx.doi.org/10.5209/rev_MESO.2014.n13.49435 
En síntesis, para que el diseño de una mediación comunicacional sea exitosa es necesario tener información sobre las necesidades y problemas que confrontan las comunidades implicadas en la gestión, conocer las redes y sistemas de comunicación con los cuales se cuentan en dichas comunidades a fin poder trabajar en red con los grupos afectados, indagar sobre los intereses de las personas o grupos, para generar la cooperación y proveer un procesos de retroalimentación que apoye la adopción de nuevas actitudes y comportamientos concertados en la búsqueda de soluciones. De allí que, el primer paso, de acuerdo con Mefalopulos y Kamlongera (2008) es realizar un diagnóstico participativo que conduzca al conocimiento del sistema social, su descripción y los liderazgos naturales, así como sus potencialidades en cuanto a la construcción de alianzas, la reciprocidad y el compromiso.

\subsection{Líneas de acción para una estrategia comunicacional con enfoque participativo}

En síntesis la revisión referencial consultada es coincidente en algunos lineamientos fundamentales relacionados con la implementación de una estrategia comunicacional de enfoque participativo, que se enumeran a continuación:

a) Articulación entre los objetivos comunicacionales y el marco referencial del proyecto.

b) Realizar un diagnóstico participativo, cuya información permita establecer una línea base sobre el estado de la situación, tanto en las condiciones ambientales y sociales de la comunidad en estudio, como en los problemas confrontados y sus posibles respuestas de solución.

c) Identificar y esquematizar los perfiles de los grupos interactivos prioritarios y los actores claves.

d) La estrategia comunicacional debe contemplar tanto el ámbito interno, referido a los investigadores y coordinadores del proyecto, como el ámbito externo, dirigido a los organismos que tienen competencia en la gestión integral de cuencas y a los grupos sociales implicados.

e) Tener objetivos claros mediante los cuales se definan las soluciones y/o productos comunicacionales.

Mediaciones Sociales, № 13, 2014, pp. 131-159. ISSN electrónico: $1989-0494$.

DOI: http://dx.doi.org/10.5209/rev_MESO.2014.n13.49435 
f) Establecer los ejes de contenidos de los temas relacionados en concordancia con la información recabada en el diagnóstico.

g) Desarrollar diseños creativos de los temas y mensajes, selección de los materiales, modos de comunicación y enfoques más adecuados.

h) Creación de espacios de articulación entre todos los actores involucrados en el proyecto, a fin de propiciar el diálogo y los consensos entre los actores participantes.

La consideración de todos estos aspectos implica un proceso de coordinación, selección y elaboración de productos comunicacionales de mayor complejidad que se van alcanzando en la medida en que se van ejecutando los procesos de participación ciudadana. En tal sentido, el proyecto de Gestión Integral de Cuencas que se viene desarrollando en las cuencas de los Ríos Pao y Unare se ha venido enriqueciendo en la medida en que se van integrando los referentes históricos con los procesos de acción comunitaria llevados a cabo por los grupos de trabajo de los sub-proyectos que conforman el proyecto global, en donde la comunicación y la información han jugado un papel importante en la mediación entre los actores claves de las comunidades involucradas y las instituciones de gobierno responsables de la gestión del desarrollo.

\section{Metodología}

La metodología de análisis del proyecto se basó en una serie de indicadores de Presión-Estado-Impacto-Respuesta (Matriz PEIR, Gráfico 1), correspondiente al proyecto GEO Ciudades (PNUMA, 2003), dirigido a evitar la degradación ambiental progresiva de los ecosistemas afectados y los procesos de recuperación ambiental de los mismos, a partir de la adopción de una nueva perspectiva conceptual sobre el medio ambiente y su importancia como soporte de vida en el planeta, capaz de fundamentar acciones de gobierno local y de las comunidades involucradas.

Mediaciones Sociales, № 13, 2014, pp. 131-159. ISSN electrónico: $1989-0494$.

DOI: http://dx.doi.org/10.5209/rev_MESO.2014.n13.49435 


\begin{tabular}{|c|c|c|c|}
\hline $\begin{array}{l}\text { Dinámicas } \\
\text { de Presión }\end{array}$ & & $\begin{array}{l}\text { Estado del } \\
\text { Ambiente }\end{array}$ & Impactos \\
\hline $\begin{array}{l}\text { Dinámica } \\
\text { demográfica } \\
\text { Dinámica } \\
\text { económica } \\
\text { Dinámica de } \\
\text { ocupación del } \\
\text { territorio }\end{array}$ & $\begin{array}{l}\text { Consumo de agua } \\
\text { Aguas residuales } \\
\text { Consumo de energía } \\
\text { Emisiones atmosféri- } \\
\text { cas } \\
\text { Residuos sólidos } \\
\text { Uso y ocupación } \\
\text { del suelo }\end{array}$ & $\begin{array}{l}\text { Aire } \\
\text { Agua } \\
\text { Suelo } \\
\text { Biodiversidad } \\
\text { Ambiente } \\
\text { Construido }\end{array}$ & $\begin{array}{l}\text { Ecosistemas } \\
\text { Calidad de vida y salud } \\
\text { humana } \\
\text { Economía } \\
\text { Urbana y rural } \\
\text { Nivel político- } \\
\text { institucional } \\
\text { Medio Ambiente }\end{array}$ \\
\hline \multicolumn{3}{|l|}{ Información } & Información \\
\hline \multicolumn{4}{|c|}{ Respuestas } \\
\hline \multicolumn{2}{|c|}{$\begin{array}{l}\text { Instrumentos Político- } \\
\text { Administrativos } \\
\text { Instrumentos Económicos } \\
\text { Instrumentos Tecnológicos }\end{array}$} & \multicolumn{2}{|c|}{$\begin{array}{l}\text { Instrumentos de Intervención Fí- } \\
\text { sica } \\
\text { Instrumentos Socioculturales, } \\
\text { Educacionales y de Comunicación } \\
\text { Pública }\end{array}$} \\
\hline
\end{tabular}

Gráfico 1. Modelo PEIR. Fuente: Metodología para la Elaboración de Informes de Geociudades. (PNUMA, 2003)

Éstos indicadores se comparan en diversos contextos, para conocer como los grupos sociales constituidos en comunidades inciden en el medio ambiente, a través de factores que presionan los ecosistemas locales dando origen a un determinado estado del ambiente con impacto sobre la calidad de vida de dichas comunidades, y provocando una serie de respuestas específicas de los gobiernos y de otros actores locales (Ver gráfico 1). Para operacionalizar la ejecución del proyecto bajo esta metodología, se conformaron cinco grupos de trabajo, cada uno de ellos encargado de un subproyecto específico, como se muestra en el Cuadro 1.

Mediaciones Sociales, № 13, 2014, pp. 131-159. ISSN electrónico: 1989-0494.

DOI: http://dx.doi.org/10.5209/rev_MESO.2014.n13.49435 
Cuadro 1.

Instituciones responsables de cada uno de los subproyectos en ejecución

\begin{tabular}{|c|c|c|c|c|}
\hline $\begin{array}{l}\text { Subproyec- } \\
\text { to } 1(\mathrm{SP} 1) \\
\text { Fundación } \\
\text { la Salle }\end{array}$ & $\begin{array}{c}\text { Subproyecto } \\
2(\mathrm{SP} 2) \\
\text { Fundación } \\
\text { Tierra Viva }\end{array}$ & $\begin{array}{c}\text { Subproyecto } \\
\text { 3(SP3) } \\
\text { Universidad } \\
\text { Pedagógica } \\
\text { Experimental } \\
\text { Libertador y } \\
\text { Universidad } \\
\text { Simón Bolívar }\end{array}$ & $\begin{array}{c}\text { Subproyecto } 4 \\
\text { (SP4) } \\
\text { Universidad } \\
\text { Carabobo y } \\
\text { Universidad } \\
\text { Nacional Ex- } \\
\text { perimental } \\
\text { Simón Rodrí- } \\
\text { guez }\end{array}$ & $\begin{array}{c}\text { Subproyecto } \\
5 \text { (SP5) } \\
\text { Universidad } \\
\text { de Carabo- } \\
\text { bo }\end{array}$ \\
\hline $\begin{array}{l}\text { Establecer la } \\
\text { línea base } \\
\text { ecológica que } \\
\text { permite } \\
\text { estimar y } \\
\text { predecir la } \\
\text { magnitud de } \\
\text { los impactos } \\
\text { en las } \\
\text { cuencas } \\
\text { seleccionadas }\end{array}$ & $\begin{array}{l}\text { Recabar la } \\
\text { información } \\
\text { inicial sobre } \\
\text { las condicio- } \\
\text { nes de vida } \\
\text { de las pobla- } \\
\text { ciones, } \\
\text { jerarquizand } \\
\text { o los } \\
\text { problemas y } \\
\text { las } \\
\text { necesidades } \\
\text { que serán } \\
\text { incorporados } \\
\text { al modelo } \\
\text { para su ajuste } \\
\text { integral. }\end{array}$ & $\begin{array}{l}\text { Tiene la res- } \\
\text { ponsabilidad } \\
\text { de promover } \\
\text { un proceso } \\
\text { educativo o de } \\
\text { transferencia } \\
\text { en las } \\
\text { comunidades } \\
\text { mediante un } \\
\text { Programa de } \\
\text { Capacitación. }\end{array}$ & $\begin{array}{l}\text { Llevar a cabo } \\
\text { el estudio so- } \\
\text { cioeconómico y } \\
\text { el desarrollo } \\
\text { endógeno para } \\
\text { el uso } \\
\text { sustentable de } \\
\text { los recursos } \\
\text { naturales y la } \\
\text { disponibilidad } \\
\text { financiera que } \\
\text { potencia dichas } \\
\text { actividades, } \\
\text { considerando } \\
\text { la integralidad } \\
\text { en la gestión } \\
\text { de la cuenca. }\end{array}$ & $\begin{array}{l}\text { Desarrollar } \\
\text { la comunica- } \\
\text { ción pública, } \\
\text { manteniendo } \\
\text { un flujo de } \\
\text { información } \\
\text { continua } \\
\text { sobre los } \\
\text { logros del } \\
\text { proyecto y } \\
\text { las dificulta- } \\
\text { des afronta- } \\
\text { das, todos } \\
\text { ellos cohe- } \\
\text { sionados } \\
\text { mediante la } \\
\text { comunica- } \\
\text { ción y el } \\
\text { trabajo en } \\
\text { red }\end{array}$ \\
\hline
\end{tabular}

\subsection{Proceso seguido en el diseño del Modelo Comunicacional}

Las acciones planificadas, en el contexto de la ejecución del proyecto, a los fines de lograr los objetivos del mismo con la intervención del uso de los medios de comunicación, se fueron diseñando progresivamente en función de los recursos disponibles, la información recabada y el marco referencial de la matriz PEIR (2003). Bajo estas premisas, inicialmente se es-

Mediaciones Sociales, N 13, 2014, pp. 131-159. ISSN electrónico: 1989-0494.

DOI: http://dx.doi.org/10.5209/rev_MESO.2014.n13.49435 
bozaron una serie de fases operativas (Indagación del contexto, diseño de la estrategia, plan de acción y evaluación) como base a la elaboración de un modelo comunicacional que se reconstruye permanentemente en la medida en que el proyecto se desarrollaba.

Es oportuno señalar que las fases se fueron cumpliendo en paralelo, ya que la ejecución del proyecto así lo exigía, por consiguiente los análisis reflexivos que se hicieron como producto del estudio realizado dieron paso a nuevos esquemas de organización donde se consideraron nuevos elementos asociados a una concepción holística que incluyó la estructura para el análisis previsto en la Matriz PEIR a partir de los cinco sub-proyectos, cuyos equipos de trabajo se comunicaban en red (Cuadro 1). Asumiendo el nombre del enlace noticioso en la página Web del Proyecto, creada con el propósito de llevar a cabo la comunicación en red, surgió el modelo comunicacional que se denominó Modelo Comunicacional INFOMIC (Flores, 2011), y tuvo como propósito integrar todas las acciones realizadas de manera sinérgica, como se muestra en el gráfico 2.

En este modelo se recogen las respuestas de la Matriz: Presión, ¿Por qué sucede esto? se señala en la base del gráfico, $\boldsymbol{E}$ stado, ¿qué está sucediendo con el medio ambiente?, esta información es la que se recoge en la línea base, o el diagnóstico ecológico ambiental, social, económico, institucional y de redes de relaciones en las comunidades, Impacto, Efectos producidos por el estado del medio ambiente, se muestra en las estimaciones, predicciones e impactos, y $\boldsymbol{R}$ espuesta, ¿qué podemos hacer y qué estamos haciendo en este momento? que se expone en el resto del gráfico. En el mismo se considera la comunicación para el desarrollo, como un acercamiento que combina métodos y procesos participantes de la comunicación con una variedad de herramientas que se extienden desde la radio rural a las tecnologías de información y de comunicación como proceso de apoyo a las acciones emprendidas por las distintas instituciones que cumplen el papel de agencias de desarrollo en las instancias locales.

Mediaciones Sociales, № 13, 2014, pp. 131-159. ISSN electrónico: 1989-0494.

DOI: http://dx.doi.org/10.5209/rev_MESO.2014.n13.49435 


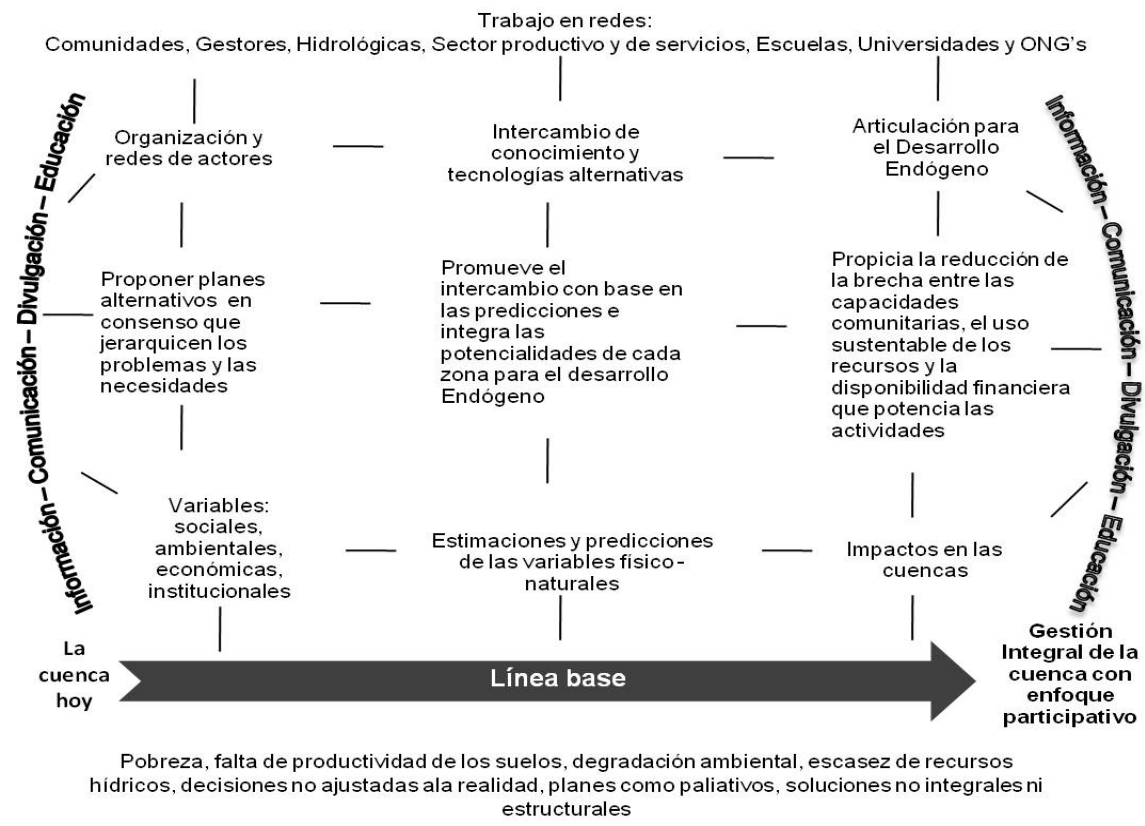

Gráfico 2. Modelo Comunicacional INFOMIC.

Fuente: Flores, M. (2011) Educación y Comunicación para el Desarrollo Sostenible en las comunidades.

Nótese, que a través de este modelo se expresa la articulación en red entre las organizaciones sociales involucradas y los ejes de acción, así como la línea base de inicio y su desplazamiento en el tiempo, hacia una mayor participación de los colectivos involucrados, no obstante, no se visualizan las etapas de concreción de los procesos que conducen a esa participación, ni cómo se integran en una estrategia global de comunicación, de allí la necesidad de reestructurar el modelo con la finalidad de complementar en uno solo todas las etapas y los procesos cumplidos.

\subsection{Modelo Comunicacional GICEP}

Retomando los lineamientos esbozados en párrafos anteriores, el modelo que se rediseñó debió contemplar el marco referencial del proyecto, sus elementos constituyentes, mostrando a su vez, la red de relaciones entre los actores claves. Y desde la perspectiva de la estrategia misma,

Mediaciones Sociales, № 13, 2014, pp. 131-159. ISSN electrónico: $1989-0494$.

DOI: http://dx.doi.org/10.5209/rev_MESO.2014.n13.49435 
«Comunicación y Mediación social para el cambio»

visibilizar los niveles de la comunicación y las fases en progreso tanto en el ámbito interno como a lo externo; así también era fundamental que expresara la transversalidad del proceso comunicacional que se llevaba a cabo. En tal sentido, surgió la necesidad de integrar en una sola estructura representacional todos los factores enunciados anteriormente y que se derivaban de la aplicación de las fases anteriores y su articulación con los actores involucrados en el proyecto general, de allí el desarrollo del Modelo Comunicacional Gestión Integral de Cuencas con Enfoque Participativo (GI$\mathrm{CEP}$ ) que se presenta a continuación (Ver gráfico 3).

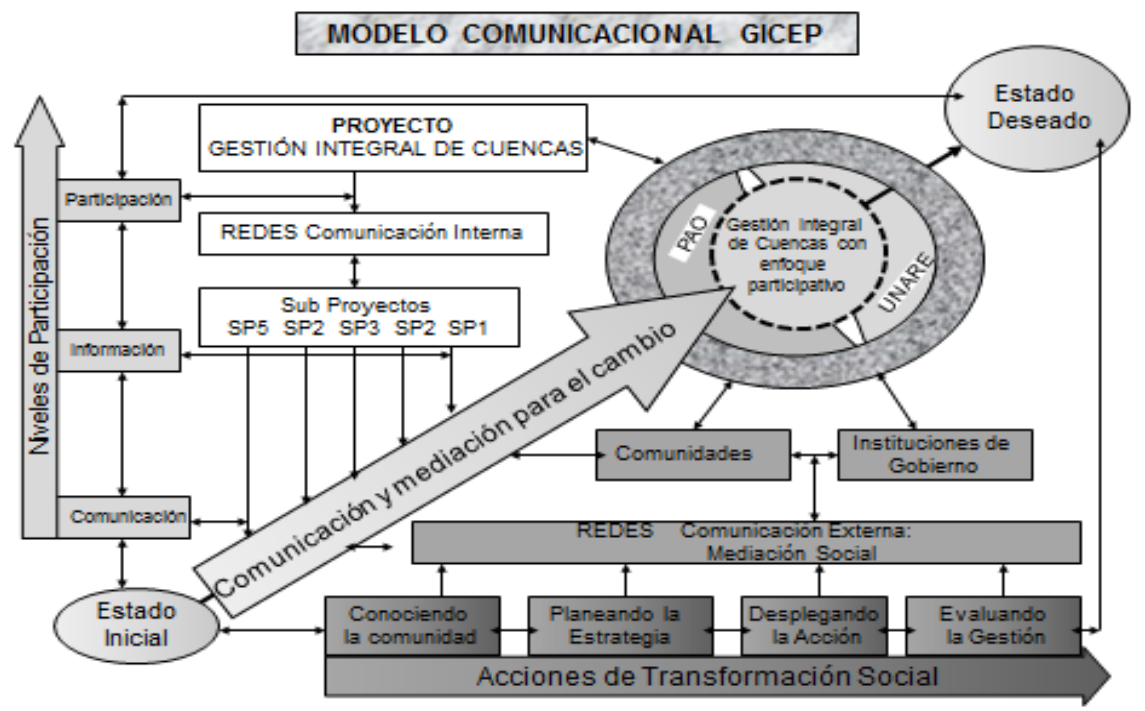

Gráfico 3. El Modelo Comunicacional GICEP

Como se aprecia en dicho modelo, la comunicación se concibe como un proceso bidireccional que orienta a todos los actores involucrados hacia dónde se quiere llegar, es decir: el estado deseado, suministrando la información necesaria para movilizar la acción de las organizaciones sociales presentes hacia el cambio y la transformación social, mediante la aplicación del modelo de Gestión Integral de la Cuenca con Enfoque Participativo. En el mismo se muestran por un lado (eje horizontal), las etapas que se vienen cumpliendo para la transformación social, a partir de un proceso de comunicación externa dirigido a poner en contacto a las comunidades y las instituciones de gobierno, que es requisito fundamental para llevar adelan-

Mediaciones Sociales, № 13, 2014, pp. 131-159. ISSN electrónico: $1989-0494$.

DOI: http://dx.doi.org/10.5209/rev_MESO.2014.n13.49435 
te un modelo de gestión integral; y por otro (eje vertical), los niveles de la comunicación que se han puesto en marcha desde las coordinaciones de los 5 sub-proyectos, para estructurar el plan de gestión y concreción de las distintas actividades planificadas en dicho proyecto. En definitiva, el estado deseado involucra la realización y evaluación de todo el proceso en todas sus etapas, hasta alcanzar su sustentabilidad en el tiempo.

En general, como se observa en el gráfico 3, cada uno de los momentos o acciones de transformación social fueron cumpliéndose en conjunción con todos los sub-proyectos, de manera que el trabajo en equipo se desplegó combinando reuniones de trabajo a lo interno del grupo y activando la participación integral de los pobladores en cada una de las comunidades participantes: Pira Pira, Palmar de Paya y Palmarote en la Cuenca del Río Pao, Municipio Libertador del Estado Carabobo; y Onoto y Granadillo en la Cuenca del Río Unare, Municipio Cajigal del Estado Anzoátegui.

\section{Recuperación de la experiencia vivida}

Seguidamente se presenta una síntesis de las actividades cumplidas en cada uno de los momentos planificados; tal como se muestra en el Cuadro 2, el desarrollo del proceso de comunicación y divulgación del proyecto de gestión de cuencas a las comunidades que hacen vida en el territorio de las cuencas en estudio, se lleva a cabo mediante diversas fases de acuerdo con cada uno de los momentos señalados.

\section{Cuadro 2.}

Momentos del proceso seguido

\begin{tabular}{|c|c|c|c|}
\hline $\begin{array}{l}\text { 1) Conociendo las } \\
\text { Comunidades }\end{array}$ & $\begin{array}{l}\text { 2) Planeando la } \\
\text { Estrategia }\end{array}$ & $\begin{array}{l}\text { 3) Desplegan- } \\
\text { do las Acciones }\end{array}$ & $\begin{array}{l}\text { 4) Evaluando } \\
\text { la Gestión }\end{array}$ \\
\hline $\begin{array}{l}\text {-Indagación del } \\
\text { contexto } \\
\text {-Diagnóstico par- } \\
\text { ticipativo } \\
\text {-Identificación de } \\
\text { grupos prioritarios } \\
\text { y organizaciones } \\
\text { institucionales }\end{array}$ & $\begin{array}{l}\text {-Diseño de la es- } \\
\text { trategia } \\
\text {-Organización de } \\
\text { la estrategia, mo- } \\
\text { dos y enfoque } \\
\text {-Eje de contenidos } \\
\text { y temas } \\
\text {-Materiales, cana- } \\
\text { les y medios de } \\
\text { comunicación }\end{array}$ & $\begin{array}{l}\text {-Gestión del } \\
\text { plan en el marco } \\
\text { referencial } \\
\text {-Activando la } \\
\text { participación } \\
\text { integral }\end{array}$ & $\begin{array}{l}\text {-Seguimiento } \\
\text { del proceso } \\
\text {-Evaluación de } \\
\text { la participación } \\
\text {-Evaluación del } \\
\text { éxito }\end{array}$ \\
\hline
\end{tabular}

Mediaciones Sociales, № 13, 2014, pp. 131-159. ISSN electrónico: 1989-0494.

DOI: http://dx.doi.org/10.5209/rev_MESO.2014.n13.49435 


\subsection{Conociendo las comunidades}

La premisa inicial que se tenía desde la perspectiva comunicacional era la de divulgar sistemáticamente los resultados obtenidos de todas las actividades realizadas en las comunidades involucradas en el proyecto, a través de estrategias y medios de difusión de información que posibilitaran la popularización del Modelo de Gestión de Cuencas, para lo cual se tomaron en consideración las características identificadas del público meta al inicio del proyecto (Arana, Flores y Díaz, 2009:1), en términos de la disponibilidad y uso de los medios de comunicación con los que contaban los pobladores, y las expectativas de comunicación de los usuarios, lo cual se logró mediante la aplicación de un instrumento de opinión semi-estructurado, que se aplicó a un grupo de informantes claves. Estos resultados mostraron que las comunidades, aunque no contaban con medios informáticos para acceder a Internet, disponían de medios de comunicación masivos como emisoras comunitarias, prensa, entre otros, mediante los cuales se les podía dar a conocer los avances del proyecto, así como otros temas fundamentales.

Una vez identificadas las situaciones problemáticas se procedió a reflexionar sobre la mejor manera de llevar la información de los resultados con la finalidad de identificar: a) sus principales preocupaciones, b) cómo veían ellos la cuenca, c) sus problemas y su motivación para la participación. Dicha información permitió organizar con mayor acierto la estrategia comunicacional, considerando ahora las situaciones problemáticas dentro de la matriz PEIR (2003), es decir: Problema-Estado-Impacto-Respuesta, como eje vertebrador de los mensajes y los temas en desarrollo; y por otra parte, configurar el plan de acción en términos de los métodos, materiales, modos y medios pertinentes a la estrategia de comunicación tanto interna como externa.

Durante el proceso de comunicación y divulgación de la información científica, se fue sensibilizando a los actores involucrados, sobre la importancia de orientar el crecimiento productivo bajo la premisa de alcanzar el desarrollo sustentable mediante criterios de ahorro e inversión, lo cual se hace factible solamente generando los cambios de actitudes necesarios para erradicar las prácticas inadecuadas de productividad que se desarrollan en el entorno de estas cuencas.

Mediaciones Sociales, № 13, 2014, pp. 131-159. ISSN electrónico: 1989-0494.

DOI: http://dx.doi.org/10.5209/rev_MESO.2014.n13.49435 


\subsection{Planeando la Estrategia Comunicacional}

En sintonía con los autores consultados (Ridder, Mostert y Wolters, 2005; Hesselink, Goldstyein, van Kemper, Garnett y Dela, 2007; Mefalopulos y Kamlongera, 2008), se definió la estrategia comunicacional tanto a nivel interno como externo, considerando:

a) el diseño de la estrategia,

b) la organización,

c) selección de modos y enfoque,

d) la elaboración de los mensajes y temas; así como

e) los materiales, canales y medios.

A continuación se presenta un esquema general que sintetiza el proceso realizado.

\section{2.a. El diseño de la estrategia}

Inicialmente se decidió que la comunicación interna estaría limitada a los investigadores adscritos a cada sub-proyecto, esto se hizo en forma continua durante todo el proyecto, a través de la página web y el correo electrónico, además reuniones mensuales y jornadas de avance. Así mismo se decidió que la comunicación externa se realizaría en dos direcciones, por una parte al público general a través de la página web, reportajes periodísticos (seis) y notas publicitarias (10) en prensa, así también micros radiales (4). Y por otra, a la comunidad involucrada mediante boletines impresos (4), calendarios (3), afiches (2), desplegables (4), entre otros.

\section{2.b. Organización de la estrategia}

La estrategia se organizó en base a las problemáticas encontradas y la formación del colectivo para dar respuestas de solución, es decir, tomando en cuenta la organización del Consejo Comunal, de manera de poderlos orientar en la elaboración de los proyectos comunitarios, lo cual formaba parte de las respuestas de solución.

Dado que el fin último de la comunicación, era que sirviera de herramienta mediacional para la transformación social de las comunidades implicadas, se optó por seleccionar el enfoque de la información para la movili-

Mediaciones Sociales, № 13, 2014, pp. 131-159. ISSN electrónico: $1989-0494$.

DOI: http://dx.doi.org/10.5209/rev_MESO.2014.n13.49435 
zación, no sólo a modo de información, sino creando un interés favorable hacia los logros del proyecto y por otro lado, crear espacios para facilitar el encuentro de los pobladores con quiénes toman las decisiones en el ámbito institucional. Esto se alcanzó mediante la ejecución de seis talleres (diagnóstico participativo), cuatro encuentros de discusión y formación (ejecución de proyectos comunales) y dos ferias del suelo y la siembra, en donde se les dio a conocer diversas experiencias sobre tipo de suelo y cultivos alternativos.
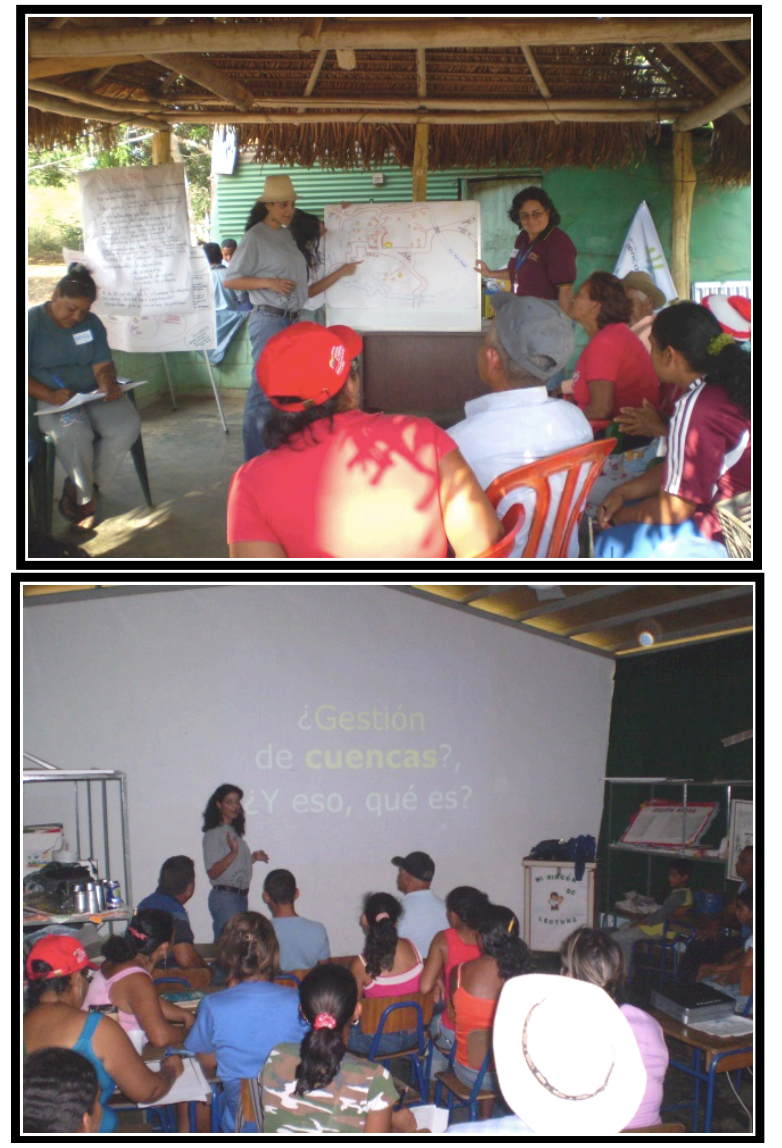

Foto 1 y 2. Talleres: Palmar de Paya y Palmarote (3 y 10 de Marzo, 2009) respectivamente en la Cuenca del Rio Pao, Municipio Libertyador, Edo. Carabobo.

Mediaciones Sociales, № 13, 2014, pp. 131-159. ISSN electrónico: 1989-0494.

DOI: http://dx.doi.org/10.5209/rev_MESO.2014.n13.49435 


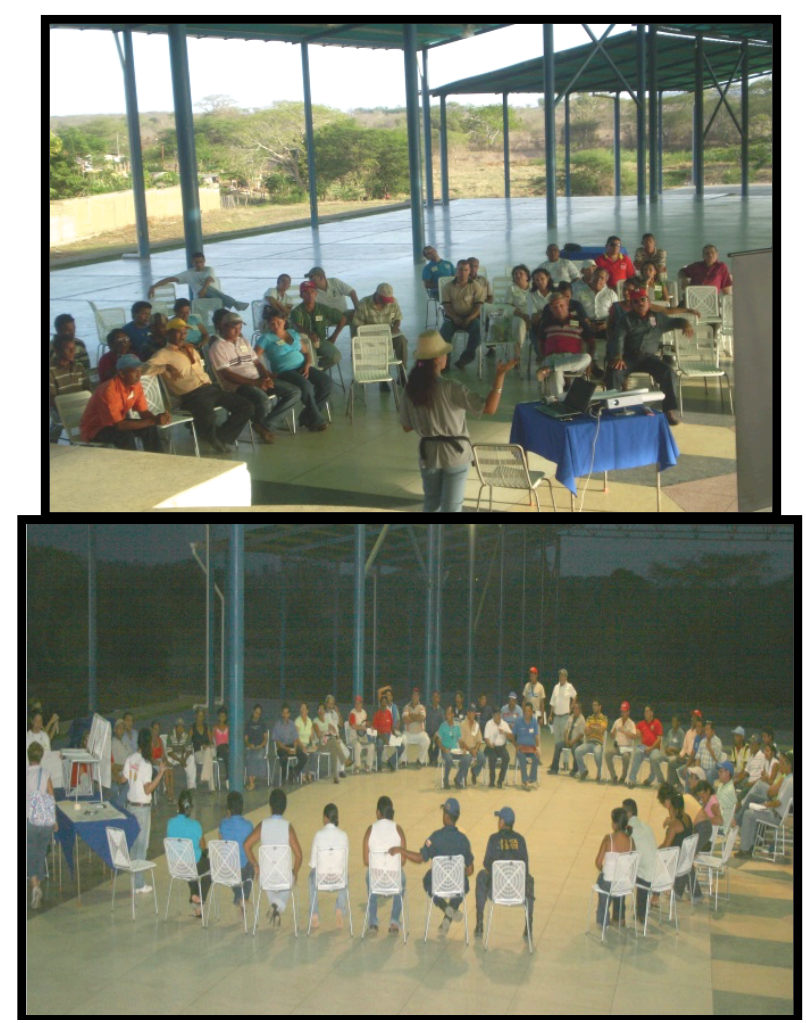

Fotos 3 y 4. Talleres: Onoto y Granadillo, (22 de Mayo; 11 de Junio, 2009) respectivamente en la Cuenca del Río Unare, Municipio Cajigal, Edo. Anzoategui.

\section{2.c. Eje de contenidos y temas}

La información estuvo vertebrada por tres conceptos fundamentales: Cuencas, Gestión y Gestión Integral, de forma que las personas comprendieran los significados asociados a estos conceptos y sus relaciones, así como también tomaran conciencia de que ellos son parte de la cuenca y de sus problemas, procurando al mismo tiempo el desarrollo de un compromiso por sus soluciones, que los condujera a la participación activa y entusiasta en los procesos de gestión integral que se desarrollaran en dicha cuenca.

Mediaciones Sociales, № 13, 2014, pp. 131-159. ISSN electrónico: 1989-0494.

DOI: http://dx.doi.org/10.5209/rev_MESO.2014.n13.49435 
En cuanto a los temas para provocar el diálogo, se trabajó sobre los problemas y las necesidades de las comunidades a fin de generar puntos de vista sobre las posibles causas y las consecuencias de un determinado problema, y como llegar a los consensos colectivos necesarios para las soluciones.

\section{2.d. Materiales, canales y medios}

Desde el inicio del proyecto se consideró llevar a cabo la información y la comunicación pública a través de diversos canales y la combinación en distintos medios, de hecho en la primera fase del mismo se creó la página web, los reportajes en prensa, los boletines impresos y electrónicos, micros radiales, artículos en revistas de investigación, participación en eventos y jornadas; en una segunda etapa, ya con un conocimiento mayor de la comunidad, se orientó la campaña comunicacional hacia la población, mediante boletines impresos, desplegables, calendarios, que contuvieran imágenes visuales con colores brillantes, pocos textos con mensajes atractivos, muchas fotos de sus pobladores en labores cotidianas, que reflejaran sus costumbres, su cultura, sus labores productivas, así como la fauna y flora características de la localidad.

\subsection{Desplegando la acción comunicacional}

Ya en una primera publicación, se reportaron los resultados de la investigación desarrollada en el año 2009 asistiendo a diversos y congresos de investigación, con la finalidad de divulgar el proyecto en las instancias académicas (Arana, Flores y Díaz, 2009; Flores, Arana y Díaz, 2011), en este caso particular se reportó la actividad realizada en el año 2010 y 2011.

\section{3.a. Gestión del plan en el marco referencial}

La gestión del plan se puso en marcha a partir de la entrega de los resultados a la comunidad de la aplicación del cuestionario para indagar sobre los grupos prioritarios, sus condiciones socioeconómicas, y definir los perfiles de los grupos interactivos para cada problema central, en cada una de las comunidades involucradas (Resultados del diagnóstico participativo); durante ese lapso se les entregó el Calendario 2010, el mismo estaba enfocado a establecer la relación ríos, cuencas y poblados, con la finalidad de ubicar en el contexto a los habitantes de estas localidades.

Mediaciones Sociales, № 13, 2014, pp. 131-159. ISSN electrónico: $1989-0494$.

DOI: http://dx.doi.org/10.5209/rev_MESO.2014.n13.49435 
Consecutivamente, se llevaron a cabo dos foros con el objeto de concretar un encuentro entre los habitantes de las comunidades y los gestores institucionales, durante la realización de los mismos se les distribuyó un desplegable, con información referida a la cuenca, pero siguiendo la matriz PEIR. Posterior a esta actividad, durante la realización de los talleres para la elaboración de los proyectos comunitarios, en Febrero de 2011, se entregaron los boletines impresos y el calendario 2011. Finalmente se cerró el ciclo con la participación de las comunidades en dos encuentros de cierre, donde se les entregó además el calendario del año 2012.

\section{3.b. Activando la participación integral}

La participación comunitaria requiere, no sólo de una voluntad política, sino de la articulación social que se genere entre los actores y los gestores a través de mecanismos de concertación, buscando siempre acciones integradoras que permitan obtener beneficios tanto en lo productivo, como en lo ambiental; considerando además la participación de todos los afectados en las decisiones, con el fin de tender a la equidad. En este sentido la comunicación y divulgación de la información se orientó en todo momento hacia el desarrollo de una visión común, promoviendo la participación activa de todas las partes interesadas con la finalidad de resolver los conflictos presentes en las comunidades, tanto en el ámbito social como ambiental.

\subsection{Evaluando la gestión}

Cuando se revisan los antecedentes relacionados a la evaluación del proceso de gestión, se hace referencia a tres aspectos fundamentales, que son: el seguimiento del proceso, la valoración de la participación y el éxito logrado, todo ello dentro de una perspectiva temporal que propone contrastar la situación actual con la situación de partida y considerando también la situación previsible (Alberich, Arnanz, Basagoiti, Belmonte, Bru y otros, 2009), mediante indicadores apoyados en los procesos de participación, según los objetivos de la comunicación y las actividades desarrolladas para tal fin. Por otra parte, se recomienda tomar en cuenta la evaluación de los contenidos en cuanto a su comprensión para que los resultados sean explicables a la luz de su contribución en cuanto al éxito logrado, así también, los procedimientos para comprender las política, sus regulaciones, los sistemas de financiamiento y apoyo (Ridder, Mostert y Wolters, 2005).

Mediaciones Sociales, № 13, 2014, pp. 131-159. ISSN electrónico: 1989-0494.

DOI: http://dx.doi.org/10.5209/rev_MESO.2014.n13.49435 
Asimismo se recomienda valorar las relaciones en términos de gestionar la colaboración, la capacidad de las redes para incrementar la inclusión y el compromiso compartido.

De manera que, la evaluación del proceso en general tiene distintos escenarios y fuentes de información, su mayor contribución en el proceso de gestión se orienta a integrar los beneficios del proyecto en la memoria de todos los actores implicados, dentro de un contexto de mejora continua, centrando su atención en el aprendizaje social, mejorar el trabajo en equipo, precisar los aspectos relevantes, compartir los hallazgos enlazados con la acción y principalmente crear una visión común para la participación activa y comprometida.

De la información recabada en las comunidades involucradas a partir de la aplicación de un instrumento de opinión, se desprendió:

a) El compromiso de los pobladores en seguir participando activamente en el desarrollo de este proyecto que los ha beneficiado en términos de su aprendizaje social, una mejor productividad y sobre todo en convivir con una mayor conciencia del entorno que comparten y de la relación que deben promover para tener una mejor calidad de vida.

b) Se evidenció también, una toma de conciencia sobre las fortalezas del trabajo en equipo, sobre la responsabilidad ante los compromisos establecidos en conjunto y por consenso, sobre todo el desarrollo del sentido de pertenencia, así como el valor de la inclusión para articular los procesos de solución de conflictos y el desarrollo de una visión común para resolver los problemas de la comunidad con ética y equidad.

c) Se asumió la importancia de la conformación de las redes para promover la participación activa de todos los interesados y su articulación con las instituciones de gobierno para incrementar los procesos de cogestión asociados a la solución de los problemas de la comunidad pero en armonía con las condiciones del ambiente para promover un desarrollo sustentable de la cuenca.

Mediaciones Sociales, № 13, 2014, pp. 131-159. ISSN electrónico: 1989-0494.

DOI: http://dx.doi.org/10.5209/rev_MESO.2014.n13.49435 
d) En cuanto a los objetivos y metas propuestas por el equipo responsable de la comunicación pública, se observó un aumento en el conocimiento y comprensión de los conceptos claves para la interiorización a nivel individual y colectivo del proyecto, su identificación con los planteamientos éticos, en términos de aprendizaje social para la transformación comunitaria y el papel que desempeña cada uno de ellos como colectivo en la protección y conservación de los recursos naturales y sociales con los que interactúan en el territorio de la cuenca.

\section{CONCLUSIONES}

Tal como se desprende de los aportes de investigaciones realizadas en esta misma línea de acción, hay un objeto común en toda la región de América Latina orientado a la conservación de las cuencas geográficas considerando la participación de los pobladores que habitan el entorno geográfico de cada una de las cuencas en estudio, ya que está demostrado por las experiencias anteriores que si no se educa y sensibiliza a estas comunidades, cualquier esfuerzo gubernamental se pierde en el tiempo, debido a que no se desarrolla el sentido de pertenencia tan necesario para involucrar a la población en la gestión integral de su propio entorno y la importancia de estos recursos naturales en el desarrollo de su calidad de vida.

Como resultado del estudio se reestructuró el modelo inicial, a un modelo tridimensional que toma en cuenta la interacción entre los grupos de trabajo, las instituciones y las comunidades involucradas en el estudio, también la incorporación de todos los procesos de gestión desarrollados por las coordinaciones de los distintos sub-proyectos, así como los actores claves involucrados y su interacción en los espacios de discusión creados con este fin, desarrollando un esquema representativo (Gráfico 3) que articula todos los aspectos fundamentales que se requieren para llevar a cabo un proceso de comunicación pública integrado, dirigido a mediar los procesos de aprendizaje social para el diálogo y la concertación entre los actores de una comunidad.

Por otra parte, se pudieron definir las principales líneas de acción de la estrategia comunicacional empleada, su mejor emplazamiento en cuanto a la articulación entre los objetivos comunicacionales y el marco referencial del proyecto, desarrollando los mejores diseños creativos de los temas

Mediaciones Sociales, № 13, 2014, pp. 131-159. ISSN electrónico: 1989-0494.

DOI: http://dx.doi.org/10.5209/rev_MESO.2014.n13.49435 
y mensajes, así como la selección de los modos de comunicación y enfoques más adecuados; pero sobre todo, creando los espacios de articulación para el diálogo entre todos los actores involucrados en el proyecto.

En otro sentido, a partir del proceso de reflexión de la experiencia vivida, se pudo constatar que la presencia en los medios ha permitido dar cuenta de la interacción Estado-universidad-comunidad propiciando el conocimiento de las cuencas en estudio y facilitando procesos de diálogo que orientaron la toma de decisiones compartidas, así como el valor de la inclusión para articular los procesos de solución de conflictos y el desarrollo de una visión común para resolver los problemas de la comunidad con ética y equidad.

Finalmente, el impacto comunicacional en las comunidades se logró por el uso de imágenes reconocidas por los pobladores en los diversos recursos comunicacionales diseñados (calendarios, desplegables, boletines) que, acompañadas de un lenguaje sencillo, se tradujo en la identificación del proyecto y el desarrollo de un sentido de pertenencia que estimuló la participación activa de los protagonistas.

En términos de su relevancia, se considera que el componente comunicacional permitió evidenciar el proceso de aprendizaje colectivo y la evolución de la conformación de la conciencia pública en la gestión integral de cuencas, sobre todo en la asunción de convivir con una mayor conciencia del entorno que comparten y de la relación que deben promover para tener una mejor calidad de vida. Asimismo, su compilación posibilita replicar la experiencia en otros contextos.

\section{Bibliografía}

Alberich, T.; Arnanz, L.; Basagoiti, M.; Belmonte, R.; Bru, P.; EsPinar, C.; García, N.; Habegger, S.; Heras, P.; Hernández, D.; Lorenzana, C.; Martín, P.; Montañez, M.; Villasante, T.; Tenze, A. (2009). Metodologías Participativas. Manual. Madrid: CIMAS. Disponible:

http://www.redcimas.org/vol.11/pdf. [consulta: 2012, sep. 10]

ARAna, A. (2008). Construcción del saber institucional. Una mirada a su complejidad. Conferencia dictada en la VI Jornada Homenaje al Profesor Universitario. 8 de Diciembre 2008. IPR El Mácaro. Turmero. Venezuela.

Mediaciones Sociales, № 13, 2014, pp. 131-159. ISSN electrónico:

$1989-0494$.

DOI: http://dx.doi.org/10.5209/rev_MESO.2014.n13.49435 
Arana, A., Flores, M. y DíAZ, E. (2009). Mercadeando la sustentabilidad Una propuesta comunicacional para la participación del colectivo. Ponencia presentada en el VI Congreso Iberoamericano de Educación Ambiental. Del 16 al 19 de septiembre de 2009. Argentina: San Clemente del Tuyú.

Arnanz, L., Belmonte, R., García, N., Garrido, F. J. Y Heras, P. (2007). Plan de Acción de la Agenda 21 Local de Jaén. Observatorio Internacional de Ciudadanía y Medio Ambiente Sostenible. CIMAS. Disponible: http://www.aytojaen.es. [Consulta: 2011 , Nov. 15]

Bustos, J.C.M. (2006). Comunicación sostenible y desarrollo humano en la sociedad de la información. Madrid: EGRAF.

Dourojeanni, A. (2010). Protocolos, lineamientos, definiciones aplicables a la gestión integrada de recursos hídricos por cuenca hidrográfica. Elaborado en apoyo a la senagua del ecuador. Disponible: http://revistavirtual.redesma.org/vol.11/pdf. [Consulta: 2011 , sep. 10]

Flores, M. (2011). «Educación y Comunicación para el Desarrollo Sostenible en las comunidades». En: Debates Ciencias Sociales. Pp. 59-78. Ediciones Universidad de Carabobo - Facultad de Ciencias Económicas y Sociales - Dirección de Investigación y Producción Intelectual. Valencia, Venezuela.

Flores, M., Arana, A. Y DíAZ, E. (2011). «Modelo Comunicacional para la gestión integral de cuencas. Una estrategia para la participación del colectivo». Revista FaCES, Vol. XXII (2) UC.

Proyecto Regional de Cooperación Técnica para la Formación en Economía y Políticas Agrarias y de Desarrollo Rural en América Latina (FODEPAL). (2004). Gestión integrada de cuencas hidrográficas. Curso a distancia. Documento Taller III Congreso Latinoamericano.

HERnÁndez ARISTU, J. (2010). «Participación ciudadana y mediación social. Una reflexión desde la práctica del asesoramiento social». Mediaciones Sociales. Semestre 2 ( $\left.\mathrm{N}^{\mathrm{o}} 7\right)$, 113-142. Madrid: Universidad Complutense de Madrid.

Hesselink, F., Goldstein, W., Paul van Kempen, P., Garnett, T. y Dela, J. (2007). La Comunicación, Educación y Conciencia Pública (CEPA). Una caja de herramientas para personas que coordinan las Estrategias y planes de acción nacionales sobre diversidad biológica. Montreal.

Mediaciones Sociales, № 13, 2014, pp. 131-159. ISSN electrónico: $1989-0494$.

DOI: http://dx.doi.org/10.5209/rev_MESO.2014.n13.49435 
Mefalopulos, P. Y Kamlongera, C. (2008). Diseño participativo para una estrategia de comunicación. Roma: Organización de las Naciones Unidas para la Agricultura y la Alimentación.

Programa de las Naciones Unidas para el Medio Ambiente (PNUMA). (2003). Metodología para la elaboración de los informes geo ciudades. Manual de aplicación. Versión 1. Disponible: http://www.pnuma.org/geociudades/pdf.s/. [Consulta 2009, jun 6]

Programa de las Naciones Unidas para el Medio Ambiente (PNUMA). (2005). Guía para la planificación estratégica sostenible local (agenda 21 local). Proyecto ciudadanía ambiental global 2005 federación latinoamericana de ciudades, municipio asociaciones de gobiernos locales (flacma).

Disponible: http://www.pnuma.org/geociudades/pdf.s/. [Consulta: 2011, febrero $16]$

República Bolivariana de Venezuela (2007-2013). Proyecto Nacional Simón Bolívar. Primer Plan Socialista -PPS- Desarrollo Económico y Social de la Nación. Caracas: autor.

Ridder, D., Mostert, E. Y Wolters, H. A. (2005). «Aprender juntos para gestionar juntos - La mejora de la participación pública en la Gestión del agua». En: Compilado, editado y diseñado por el equipo HarmoniCOP. Dentro del programa temático Energía, Medioambiente y Desarrollo Sostenible. (1998-2002 / No contrato EVK1-CT-2002-00120).

\section{PARA CITAR ESTE TRABAJO EN BIBLIOGRAFÍAS:}

FLORES, María Ángela; ARANA, Aracelis; DÍAZ DE MARIÑO, Esmeya (2014): "Comunicación y Mediación social para el cambio. Proyecto Gestión Integral de Cuencas con enfoque participativo. Casos: Ríos Pao y Unare", Mediaciones Sociales. Revista de Ciencias Sociales y de la Comunicación, $\mathrm{n}^{\circ}$ 13, pp. 131-159. DOI: $\mathrm{http} / / / \mathrm{dx}$. doi.org/10.5209/rev_MESO.2014.n13.49435

RECIBIDO: 21 de julio de 2013.

ACEPTADO: 11 de junio de 2014.

Mediaciones Sociales, № 13, 2014, pp. 131-159. ISSN electrónico: $1989-0494$.

DOI: http://dx.doi.org/10.5209/rev_MESO.2014.n13.49435 


\section{El autor}

(*) María Ángela Flores Páez, Profesora Asociado, Universidad de Carabobo Facultad de Ciencias Económicas y Sociales. Miembro del Grupo de Investigación en Política e Instituciones. Líneas de investigación Estudios de Gobierno Educación y Desarrollo Sustentable. Licenciada en Educación, Mención Matemática (UC). Especialista en Gerencia Pública, Mención Descentralización (UFT). Magister en Ciencia Política, mención Gerencia Pública, (UC) Doctoranda del Programa Doctoral en Innovaciones Educativas (UNEFA). Av. Salvador Allende Edificio Anexo Faces P.B. Bárbula, Municipio Naguanagua. Estado Carabobo Teléfono (0241)2056307. mariangelafp@hotmail.com; mflores@uc.edu.ve

${ }^{(* *)}$ Aracelis Arana Díaz, Profesora Asociado e Investigadora en UPEL Maracay. Profesora de Biología y Ciencia General (UPEL-IPC), Magister en Educación mención Planificación Cunicular. Doctora en Educación (UPEL IPC, 2005). Miembro del Centro de Investigaciones Educacionales Paradigma (CIEP), Coordinadora Línea de Investigación Innovaciones didácticas y Evaluación. Dirección: Av. Principal Las Delicias antiguo Parque de Ferias, Las Delicias, Maracay, Estado Aragua. Teléfono (0243)2417866. aracelisarana3@gmail.com.

${ }^{(* * *)}$ Esmeya Díaz de Mariño, Docente-Investigador Asociado del Instituto de Investigaciones de la Facultad de Ciencias Económicas y Sociales de la Universidad de Carabobo (INFACES-UC). Profesora de Biología y Ciencia General (UPEL-IPC) Magíster en Ecología IVIC. Doctora en Ciencias Biológicas (UAM - España). Línea de investigación: Desarrollo sustentable y Educación para la Sustentabilidad. Av. Salvador Allende Edificio Anexo Faces P. B Bárbula Municipio Naguanagua. Estado Carabobo Teléfono (0241) 2056370. esmeyadiazm@gmail.com ecdiaz@uc.edu.ve

Mediaciones Sociales, № 13, 2014, pp. 131-159. ISSN electrónico: 1989-0494. 